Aus der chirurgischen Klinik der Universität Leipzig. (Direktor: Geh.-Rat Professor Dr. Tre ndele n burg.)

\title{
Über Zwerchfellverletzungen und ihre operative Behandlung.
}

Von Dr. Wilhelm Wolf, K. S. Oberarzt, kommandiert zur Klinik.

Einen Fall von $Z$ werchfellverletzung hatte ich im Sommer I909 in hiesiger Klinik zu operieren Gelegenheit, der sowohl hinsichtlich der eigenartigen Ätiologie, als auch in bezug auf die Komplikationen des Verlaufs mancherlei interessantes bot, so daß er mir der Veröffentlichung wert erscheint.

Es handelte sich um einen 25 jährigen Drechsler Albert K., Mitglied eines Leipziger Ruderklubs. Der Ruderklub hatte am 29. VII. Ig09 abends ein Wettfahren auf der Pleiße veranstaltet. Dabei war ein von hinten angeschossen kommendes, mit eiserner Spitze versehenes Rennboot, das ein vor ihm fahrendes, an dessen Heck unser Verletzter als Steuermann saß, überholen wollte, infolge falscher Steuerung auf das vordere aufgerannt und hatte dessen Steuermann mit seinem Bug buchstäblich aufgespießt. Hinzugerufene Samariter befreiten den Mann aus seiner qualvollen Lage. Mit einem Notverband versehen wurde er mit tunlichster Beschleunigung in die Klinik eingeliefert.

Befund: Ausgebluteter, nur mühsam atmender Patient, der vollständig kollabiert ist und keinerlei Auskunft über den Hergang des Unfalls zu geben vermag. Elender, beschleunigter Puls. Nach Abnahme des die Brust bedeckenden Notverbandes, der außer mit Blut stark mit einer grünlich-bräunlichen Flüssigkeit, anscheinend Magensaft, durchtränkt ist, sieht man auf der linken Rückenseite, die Skapularlinie schräg kreuzend, eine ca. Io $\mathrm{cm}$ lange, weit klaffende Wunde mit unregelmäßig gerissenen und stark gequetschten Rändern. Aus der Wunde hängen große verschmutzte Muskelfetzen heraus. In der Tiefe liegt die eingedrückte II. und I2. Rippe frei, zwischen denen ein klaffender Spalt entstanden ist, gegen den die freiliegende Lunge mit ihrem stark gequetschten unteren Rand bei der Atmung rhythmisch anschlägt. Unter schlürfendem Geräusch tritt die Luft durch die große Pleurawunde ein und aus. 
Operation (Dr. W o lf): Chloroformnarkose. Da die Pleurahöhle bereits ausgiebig eröffnet ist, braucht die Wunde nicht erweitert zu werden. Nach Emporschlagen der teilweise kollabierten Lunge durch die Hand des Assistenten sieht man am Boden der Pleurahöhle Milz und Magen, zwischen denen Netz sich hervordrängt, zutage liegen, die durch eine lange, fast die ganze $Z$ werchfellkuppel von vorn nach hinten schräg durchsetzende Rißwunde mit stark zerfetzten Rändern vorquellen. Nach möglichster Austupfung des reichlichen, die Pleurahöhle erfüllenden Blutes (ca. 1/2 Liter), das mit Mageninhalt vermengt ist, bemerkt man an der vorliegenden hinteren Magenwand eine ca. pfenniggroße Perforation mit unregelmäßigem, stark suggiliertem Rande, eine zweite, ebenso beschaffene Wunde findet sich etwas weiter vorn in der Magenwand. Vernähung der Magenwunden, Reposition der prolabierten Baucheingeweide ins Abdomen, nachdem diese im übrigen unverletzt befunden worden sind, Naht des zerrissenen Zwerchfells, wobei das Nähen in der Tiefe sehr große Schwierigkeiten bereitet. In Anbetracht der starken Verunreinigung der Pleura werden 2 Drains in diese eingeschoben, im übrigen wird die Wunde, deren Ränder umschnitten und geglättet worden sind, vernäht. Subkutane Kochsalzinfusion.

30. VII. Heute abend Temperatur 38,2. Reichliche Absonderung aus der Thoraxwunde. Ziemliche Atembeklemmung. Ausgebreitetes subkutanes Emphysem im Bereich der linken Brust- und Bauchhälfte. Abdomen im übrigen weich, nicht druckempfindlich.

3I. VII. Auf Einlauf erfolgt Stuhl gleichzeitig mit Luftabgang. Die Wundumgebung ist entzündlich gerötet. Ziemlich starke Atemnot.

I. VIII. Wunde jaucht sehr stark. Erisypelatöse Entzündung in der Umgebung. Epidermis teilweise maceriert. Patient ist ziemlich verfallen. Nimmt heute zum erstenmal etwas Schleimsuppe, nachdem er bisher nur schwarzen Tee bekommen hat. Puls leidlich kräftig. Subkutanes Emphysem besteht unvermindert.

6. VIII. Die Abendtemperaturen halten sich auf ca. 38,0. Die Wundumgebung ist noch hochrot. Verhaltung unterhalb der Wunde. Hierselbst Gegenincision unter Lokalanästhesie, um dem jauchigen Wundsekret Abfluß zu verschaffen. Dre Atemnot bessert sich in demselben Maße wie das Hautemphysem zurückgeht. Der Leib ist weich, nirgends druckempfindlich. Stuhl jeden 2. Tag auf Einlauf. Seit gestern erhält Patient vorsichtig etwas festere Nahrung.

7. VIII. Die teilweise vereiterten Nähte werden entfernt. Die große Wunde ist zu etwa $2 / 3$ vernarbt. 2 mal täglich Spülung der jauchenden Pleura mit Kalium hypermangan.

Ir. VIII. Das Allgemeinbefinden hat sich sehr gebessert. Patient ißt und trinkt mit Appetit. Die Jauchung aus der Pleura läßt nach. Dafür wird sehr reichlich dünnflüssiger, weniger riechender Eiter abgesondert. 
I5. VIII. Wundumgebung reizlos. Immer noch reichliche Eiterabsonderung aus der Pleurawunde. Das Hautemphysem ist gänzlich geschwunden. Gestern abend war die Temperatur ganz normal. Das Befinden des Patienten ist ein recht gutes.

I. IX. Die Eiterung ist noch immer sehr stark. Im Bereich der bereits vernarbten, in der Richtung der Rippen verlaufenden Hauptwunde bestehen noch zahlreiche eiternde Fisteln neben der großen Pleuraöffnung, von denen aus die Sonde auf rauhen Knochen führt. Temperatur dauernd normal. Doch erholt sich Patient nicht recht,

Io. IX. Patient ist noch sehr abgemagert. Die große Pleurawunde schließt sich durch Granulationen von der Tiefe aus, so daß das noch liegende große Drain bereits mehrfach gekürzt werden mußte. Patient wird sehr von starken Hustenanfällen geplagt, die zuweilen im Anschluß an den Verbandwechsel, zuweilen auch spontan auftreten und erst wieder vorübergehen wenn Patient nach starkem Würgen reichlich eitrigen Schleim expektoriert hat. Das Ausgehustete riecht nicht

I4. IX. Gestern abend Temperatur 38,o. Da Patient immer mehr abmagert wird heute eine nochmalige genauere Untersuchung in Narkose vorgenommen. Von der granulierenden Pleurawunde aus gelangt eine nach oben subkutan in eine dort bestehende Fistel eingeführte Sonde in eine große Höhle. Um dieselbe freizulegen wird ein ca. $6 \mathrm{~cm}$ langes Stück der nächsthöheren Rippe reseziert. Bei Eröffnung der Pleura entleert sich zunächst bestialisch riechende Luft. Beim Aufsitzen fließt etwa 1/2 Liter stinkende bräunliche Jauche, vermischt mit gangränösen Lungenfetzen, ab. Drainage der Höhle.

20. IX. Unter regelmäßigen Spülungen mit Kalium hypermangan. läßt der Geruch des Pleurasekrets nach, das Allgemeinbefinden bessert. sich etwas.

27. IX. Markstückgroßer Dekubitaldefekt am Kreuzbein.

I2. X. Aus der unterminierten Haut in der Umgebung der Hauptwunden entleert sich durch Incision reichlich Eiter. Patient magert immer mehr ab.

29. X. Sekretion aus der Empyemhöhle immer noch ziemlich reichlich und sehr übelriechend. In den letzten Tagen wieder abendliche Temperatursteigerungen über 38 ,o. Knöchelödem. Schwache Herztätigkeit. Digalen subkutan. Das Abdomen ist etwas aufgetrieben, besonders im Bereich des Epigastriums. Hier klagt Patient auch über ein Gefühl der Völle und Druckempfindlichkeit. Urin frei von Eiweiß.

30. X. Heute nachmittag hochgradiger Kollaps. Bewußtlosigkeit, mühsam schnappende Atmung, Cyanose. Nach wiederholten Kampferinjektionen erholt sich Patient wieder.

4. XI. In den letzten Tagen zunehmende Schwäche bei großer Appetitlosigkeit. Extreme Abmagerung. Zunehmende Ödeme. Heute Exitus letalis. 
Sektionsbefund ${ }^{1}$ ) (Obduzent Dr. V e r s é): Brusthöhle: Zwerchfellstand beiderseits 5. Rippe. Die linke Pleurahöhle ist zum großen Teil mit Luft gefüllt. Von der äußeren Fistelöffnung aus gelangt man direkt in die Pleurahöhle hinein. Die linke Lunge liegt an der medialen Seite der letzteren. Die Pleura ist bedeckt mit geröteten Granulationen, die eine eitrige Schicht tragen. In den hinteren Abschnitten der Pleurahöhle findet sich etwas eitrige Flüssigkeit. In der atelektatischen linken Lunge sind unten größere käsige Knötchen vorhanden neben einer kleinen Höhle, die ein kleines schalenförmiges gezacktes Knochenstück enthält, das von kleinen gelblichen Brörkeln umgeben ist. Diese kleine, am unteren Rand gelegene Höhle hängt direkt mit einem stark geröteten Bronchus zusammen. Das übrige Parenchym ist sehr feucht und vollständig luftleer, die erwähnte Aussaat von Knötchen, die in der Form von Strängen angeordnet sind, beschränkt sich auf die Umgebung der beschriebenen Höhle. Im obersten Teil des Mediastinums findet sich eine mittelgroße verka!kte Drüse, am Lungenhilus sitzen ziemlich geschwollene anthrakotische Drüsen.

Bauchhöhle: Zwischen dem linken Leberlappen und dem Zwerchfell bestehen einige Verwachsungen. Aus dem linken Leberlappen heraus, der anscheinend eine Höhle enthält, quillt eitrige Flüssigkeit. Das große Netz ist ebenfalls an der linken Zwerchfellkuppe adhärent. Auch der Fundus des Magens ist mit dem Zwerchfell verwachsen. Die Milz ist ganz in Verwachsungen eingebettet, hat graurötliche Farbe. In den Verwachsungen unterhalb des Zwerchfells zwischen Milz und Fundus befinden sich noch gelbliche, offenbar aus verfettetem Granılationsgewebe bestehende Einlagerungen. Außen an der großen Kurvatur sitzt eine taubeneigroße Vorwölbung, die von Verwachsungen des Netzes umgeben wird, eine ziemlich feste Beschaffenheit hat und graue Färbung zeigt. Nach dem Aufschneiden des Magens ist zu bemerken, daß bei leichtem Druck auf die Vorwölbung dicker gelblicher Eiter durch eine kleine fistulöse Offnung der Schleimhaut hervorquillt. Leber, Magen, Milz und linke Lunge werden zusammen mit den Halsorganen herausgenommen. Beim weiteren Aufschneiden des Magens wird oben am Fundus, direkt an das Diaphragma anstoßend, eine streifenförmige, graurote $2 \frac{1}{2} \mathrm{~cm}$ lange Narbe erkennbar. Ringsherum finden sich fleckige Pigmentierungen der gequollenen Magenschleimhaut. Aus der beschriebenen, an der großen Kurvatur bezw. an der hinteren Magenwand befindlichen Vorwölbung entleert sich beim Einschneiden dünnflüssiger Eiter. Die Abszeßhöhle ist etwa haselnußgroß, von grauschwärzlichem Gewebe eingeschlossen. Im linken Leberlappen findet sich eine sowohl an der Oberfläche als auch an der Leberunterfläche deutlich ausgesprochene Vorwölbung, aus der sich nach Eröffnung stinkender graugrünlicher Eiter entleert. Diese Eiterhöhle

I) Das Sektionsprotokoll ist nur auszugsweise wiedergegeben. Soweit die cinzelnen Organe nicht crwähnt sind, boten sie keine Besonderheiten. 
hat reichlich die Größe eines großen Apfels und reicht etwas noch in den rechten Leberlappen hinein. An ihrer etwas unregelmäßigen Innenfläche sitzen dicke, graugelblich-grünliche Auflagerungen. Sie ist von einem derben schiefriggrauen Gewebe umgeben und hängt mit mehreren kleineren, nach hinten und lateral im linken Lappen gelegenen, anscheinend frischeren Höhlen zusammen, die $z$. T. eine mehr schmutziggraue Färbung haben.

Was nun zunächst die Ätiologie unserer Zwerchfellverletzung betrifft, so dürfte diese ganz einzig in ihrer Art dastehen. Die in der Literatur beschriebenen Verletzungen des Diaphragma sind, soweit sie p e r k u t a n e r Natur sind, zunächst Stichverletzungen, in zweiter Linie Schußverletzungen. Dazu kommen die subkutanen Zwerchfellverletzungen, die zumeist Rupturen infolge heftiger Kontusion des Thorax darstellen. Letztere machen nach $\mathrm{R}$ i e d i n g e ${ }^{1}$ ) etwa $2 / 3$ aller Zwerchfellverletzungen aus.

In unserem Falle liegt eine perkuta n e Verletzung vor, aber eine solche so gigantischer Natur, wie sie noch nicht beobachtet sein dürfte. Die ganze eiserne Spitze des Rennbootes war dem Verletzten in den Thorax eingedrungen und hatte sich so fest eingebohrt, daß sie nach Erzählung der Augenzeugen des Unfalls erst gewaltsam herausgezogen werden mußte. Man könnte ja zunächst daran denken, daß es durch den starken Stoß zu einer Ruptur des Zwerchfells schon vor dem Eindringen der Bootsspitze in den Thorax nach Art der erwähnten subkutanen Rupturen gekommen wäre, und daß die Eisenspitze selbst gar nicht so tief vorgedrungen wäre, gegen diese Annahme spricht jedoch die doppelte Durchbohrung der Magenwand, die nur auf direktem Wege zustande gekommen sein kann.

Die Zwerchfellverletzungen sind zwar an sich ziemlich häufig, wahrscheinlich noch häufiger, als man gemeinhin annimmt, da sicher eine große Anzahl kleiner Zwerchfellwunden von selbst heilt, aber die Zahl der operativ beha ndelte $\mathbf{n} Z$ werchfellverletzungen ist eine verhältnismäßig noch kleine. Die letzte große Sammelstatistik von Sute ${ }^{2}$ ), aus dem Jahre 1905 berichtet insgesamt von 79 so behandelten Zwerchfellwunden.

I) von Bergmann u. von Bruns, Handbuch der praktischen Chirurgie. Stuttgart I907.

2) Beiträge zur klin. Chir. 1905, Bd. 46 und 47 . 
Seitdem habe ich in der mir zugänglichen Literatur noch I3 Fälle veröffentlicht gefunden, die ich unten zusammengestellt habe, und denen ich noch einen in unserer Klinik im Jahre I908 operierten hinzufügen möchte, so daß also im ganzen bisher, inkl. unserem an erster Stelle beschriebenem Fall, 94 mal das verletzte Zwerchfell operativ behandelt wurde.

Zunächst folge noch in Kürze die Krankengeschichte des zweiten einschlägigen Falles aus unserer Klinik.

23 jähriger Bahnarbeiter geriet am 26. XII. Igo8 in eine Messerstecherei. Wurde schwerverletzt in die Klinik eingeliefert.

Befund: Jammernder Patient von blasser, cyanotischer Gesichtsfarbe. Sehr frequente, mühsame Atmung. Puls verhältnismäßig gut, ca. 80 -90. Im 6 . Interkostalraum rechts, etwas nach innen von der Mamillarlinie, sieht man eine ca. $2 \mathrm{~cm}$ lange klaffende Stichwunde, durch die fortwährend die Luft weithin hörbar hindurchpfeift. Beim Aufsetzen stürzt ein großer Schwall Blutes aus der Wunde heraus. Über der rechten Lunge ist kein Atemgeräusch hörbar, hinten unten 4 Querfinger breit Dämpfung. Über der linken Lunge ist das Atemgeräusch sehr leise. Die Herztöne sind leise aber rein. Die Leberdämpfung ist deutlich. Die Bauchdecken sind gespannt, aber nicht druckempfindlich, auch spontan bestehen keine Leibschmerzen.

Operation (Dr. S i e ver s): Chloroform-Äthernarkose. Ausgiebige Erweiterung der Interkostalwunde. Dabei zeigt sich, daß der 7 . Rippenknorpel von dem verletzenden Instrument durchtrennt ist. Direkt gegenüber der Wunde fühlt die eingeführte Hand in der Zwerchfellkuppel eine größere Zerreißung, aus der die Blutung anscheinend stammt. Nach Herstellung genügender Übersicht durch Auseinanderhalten der Wundränder mittels Wundhaken erkennt man einen breiten Defekt im Zwerchfell, dicht am Herzboden, aus dem hellrotes Blut vorquillt. Der Defekt wird durch 2 Katgutnähte geschlossen, worauf die Blutung steht. Austupfen der ziemlich reichlichen Blutmenge aus der rechten Pleurahöhle, vollkommener Verschluß der Thoraxwunde mit Katgut und Seide ohne Drainage. Kochsalzeinlauf per rectun.

27. XII. Patient hat sich erholt. Temperatur normal. Puls verhältnismäßig kräftig.

3. I. Wunde per primam geheilt. Entfernung der Nähte.

Io. I. Über der rechten Lunge hinten unten handbreite Dämpfung (Pleuraexsudat). Hierselbst abgeschwächtes Atemgeräusch.

20. I. Das Pleuraexsudat ist im Zurückgehen begriffen. Die Körpertemperatur ist dauernd normal.

28. I. Die Dämpfung ist ganz geschwunden. Die rechte Lunge atmet wieder ausgiebig mit.

3I. I. Geheilt entlassen. 
Sicher wird in zahlreichen Fällen von Zwerchfellverletzungen die Diagnose nicht gestellt, sonst könnte man die geringe Zahl der ausgeführten $Z$ werchfellsuturen ${ }^{1}$ ) nicht verstehen. Denn man kann nicht annehmen, daß etwa des öftern von Chirurgen, trotzdem die Diagnose feststeht, konservativ verfahren würde in der Hoffnung, daß ja solche Wunden auch spontan heilen können, wie für gewisse Fälle, nämlich für diejenigen Wunden, die in der Richtung der Muskelfaserung liegen, von Repet t ${ }^{2}$ ) experimentell nachgewiesen ist.

Dafür sind doch die eventuell aus einer unbehandelten $Z$ werchfellwunde entstehenden Nachteile zu große. Ist doch nachgewiesen, da $B$ zuweilen erst nach vielen Jahren Patienten an den Folgen ihrer $Z$ werchfellwunde zugrunde gingen, bei denen sich im Anschluß an das Trauma eine Hernie entwickelt hatte, die nun durch Inkarzeration plötzlich verhängnisvoll wurde. In der Arbeit $\mathrm{L}$ a $\mathrm{ch}$ e $\mathrm{r}^{3}$ ) über Zwerchfellhernien finden sich 9 Fälle verzeichnet, in denen der Tod an plötzlich einsetzender Inkarzeration im 2. bis 5. Jahre nach der Verletzung erfolgte, 3 starben im 5. bis Io. Jahre, 3 vom Io. bis 20 . Jahre, einer erst nach 22 Jahren.

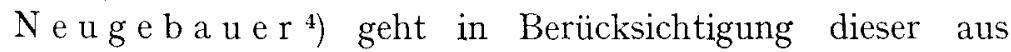
übersehenen Zwerchfellverletzungen eventuell erwachsenden Gefahren soweit, daß er bei jeder perforierenden Verletzung des unteren Abschnittes der 1 i n $\mathrm{k}$ e $\mathrm{n}$ Thoraxhälfte die Laparotomie fordert, um von unten her das Zwerchfell auf eine Wunde hin abtasten zu können. Er hält diesen Eingriff für ebenso notwendig wie die Laparotomie bei Bauchverletzungen zur Absuchung der Eingeweide. Bei den rechts sitzenden Zwerchfellwunden ist bekanntlich die Gefahr der Entwicklung einer Hernie keine große, da sich hier die massige Leber als schützender Wall vor die Wunde legt.

Es ist vielmehr, wie bereits erwähnt, die geringe Zahl der

I) Abgesehen von wenigen Ausnahmefällen bestand die operative Behaydlung der Zwerchfellwunden im Verschluß durch Naht.

2) Repetto, Heridas del diaphragma à travès del seno costo-diaphragmatico. Buenos Aires. J. Penser 1894. Zit. nach Suter 1. c.

3) Deutsches Archiv für klin. Medizin I880, Bd. 27.

4) Archiv f. klin. Chir. 1904, Bd. 73 . 
ausgeführten Zwerchfellnähte aus dem Umstand zu erklären, da $B$ die $D$ i a g $n$ os e einer Zwerchfellverletzung recht schwierig sein kann und nicht selten unmöglich zu stellen ist.

Besonders schwer ist sie häufig bei den $\mathrm{subkutanen}$ Verletzungen, den Rupturen, zu stellen, die durch Einwirkung einer plötzlich auftretenden, großen äußeren Gewalt zustande kommen, bei denen es zu einer heftigen Kompression der Bauchhöhle oder der Brusthöhle kommt, die zum Platzen des Zwerchfells, als einer besonders schwachen Stelle der Wandung des Hohlraumes, führt. Wir sprechen nicht von den Fällen, in denen die Wunden im Zwerchfell so groß sind, daß die Baucheingeweide in die Brusthöhle prolabieren, so daß wir oberhalb des Zwerchfells tympanitischen Darm- oder Magenschall haben, womöglich nach Anfüllung des Magens mit Flüssigkeit Plätschergeräusch in der Brusthöhle wahrnehmen, bei denen an sich schon das eingesunkene Abdomen gegenüber dem ausgedehnten Thorax in Verbindung mit der durch die Kompression der Lungen seitens der verlagerten Abdominalorgane verursachten Dyspnoe auffällt, sondern von den kleineren Wunden, die aber nicht selten eben durch ihre verhältnismäßige Kleinheit, später zur Inkarzeration einer aus ihnen entstandenen Hernie führen können.

Die hier vielleicht vorhandenen Symptome, Schmerzen in der Gegend des Zwerchfells, besonders beim Tiefatmen und beim Husten, Ubelkeit, Aufstoßen, sind $\mathrm{zu}$ wenig charakteristisch als daß sie uns die Diagnose einer Zwerchfellverletzung stellen ließen, und das sogenannte ,sardonische Lächeln“ des französischen Chirurgen P e r e y ${ }^{1}$ ), das dieser auf den Gesichtszügen gefallener Soldaten bemerkt haben will, bei denen hinterher gelegentlich der Autopsie sich eine Zwerchfellverletzung vorfand, so daB er glaubte, daraufhin die Diagnose stellen zu können, konnte von späteren Autoren, wie $\mathrm{S} \mathrm{u} \mathrm{t}$ e r betont, nicht wieder konstatiert werden.

Leichter wird ja im allgemeinen die Diagnose der perku ta ne n Verletzungen des Diaphragma sein. Besonders wenn die Wunde im Bereich des Thorax liegt und Eingeweide des Abdomens prolabiert sind kann an der Diagnose kein Zweifel sein. Zuweilen

I) Zit. nach Suter 1. c. 
ragte Netz aus der äußeren Thoraxwunde heraus. Sorrent i n $o^{1}$ ) berichtet über einen Fall, in dem der ganze Magen durch die äußere Wunde vorgefallen war.

In manchen Fällen, wie in unserem ersten Falle, läßt der aus der Thoraxwunde ausfließende Mageninhalt erkennen, daß nicht nur das Zwerchfell verletzt ist, sondern auch der darunter liegende Magen. In dem Falle $\mathrm{Ne} \mathrm{ug} \mathrm{e} \mathrm{b} \mathrm{a} \mathrm{u} \mathrm{e} \mathrm{r}{ }^{2}$ ) entströmte der Thoraxwunde bei jedem Atemzug Alkoholgeruch. Der Alkoholduft entstamnite dem Magen. Der Verletzte hatte, kurz bevor er verwundet wurde, 3 Liter Bier getrunken. Auch Severean $u^{3}$ ) und $\mathrm{Zawadz} \mathrm{ki}$ ) berichten von Fällen, in denen Mageninhalt aus der Thoraxwunde ausfloß.

Uberhaupt sind Nebenverletzungen bei Zwerchfellwunden ein sehr häufiger Befund, was bei den perkutanen Verletzungen wenigstens sehr natürlich ist. Denn wie wir sahen, sind die häufigsten Ursachen für die Zwerchfellwunden Stich- und Schußverletzungen. Da nun solche Wunden in der Regel schräg verlaufen werden, entweder von unten nach oben oder umgekehrt, so wäre es nicht recht verständlich, warum das verletzende Instrument bzw. die perforierende Kugel nur das flache Zwerchfell und nicht auch die Organe oberhalb oder unterhalb desselben treffen sollte. Bei dieser Gelegenheit möchte ich einer interessanten Beobachtung Erwähnung tun, die der Mailänder Chirurg Ross ${ }^{5}$ ) gemacht hat. Bekanntlich ist Italien das Land der Messerhelden. Nach $\mathrm{R} o s \mathrm{~s}$ is Erfahrung führen nun die römischen und neapolitaner Messerhelden die Klinge an der Kleinfingerseite und stechen demgemäß von oben nach unten, während die Milanesen mit größerer Vorliebe das Messer mit der vollen Faust (Klinge an der Daumenseite vorragend) halten und die Verletzungen von unten nach oben zufügen.

S u ter hat in 36,9 Proz. seiner Fälle Nebenverletzungen feststellen können. Der Hänfigkeit nach geordnet standen im

1) Riforma med. i895, Nr. 82; Ref. Zentrabl. f. Chir. 1895, Nr. 26.

2) Archiv f. klin. Chir. I904 Bd. 73 .

3) Congrès français de chir. 1893; zit. nach Suter 1. c.

4) Kronika lekarska I902, Nr. I6-ig. Ref. Zentralbl. f. Chir. I903, S. 296.

5) Contributo alla cura della ferite diaframmatiche (Clinica chirurgica Igo5,

Nr. 4). Ref. Zentralbl. f. Chir. Igof, S. jos. 
Vordergrund die Mitverletzungen des Magens, dann folgten die der Leber, der Lunge, des Colon, der Milz, der Niere. . Auch einige Fälle, in denen mehrere Organe gleichzeitig mitverletzt sind, sind beschrieben. In unserem $x$. Fall war, wie wir sahen, der Magen 2 mal verletzt; in dem 2. Fall bestand wahrscheinlich eine Mitverletzung der Leber, wofür die in der Pleurahöhle befindliche reichliche Blutmenge spricht, die kaum aus der Zwerchfellwunde allein sich erklären ließe. Bei der Naht der Zwerchfellwunde wurde die unmittelbar darunter sitzende Leberwunde mit vernäht, wodurch die Blutung zum Stehen kam.

Wesentlich beeinträchtigt wird durch solche Nebenverletzungen natürlich die Prog nos e der operierten Zwerchfellverletzungen. Während $\mathrm{Suter}$ für die von ihm zusammengestellten, operativ behandelten $Z$ werchfellwunden eine Mortalität von 12,3 Proz. berechnete, wenn er alle Fälle gleichmäßig in Rechnung zog, ob sie nun durch Nebenverletzungen anderer Organe kompliziert waren oder nicht, muß er zugeben, daß die Mortalität sofort doppelt so groß ist (25 Proz.), wenn die mit Verletzungen anderer Organe einhergehenden Zwerchfellwunden für sich allein der Berechnung zugrunde gelegt wurden. Außerdem muß wohl bei der Berechnung der Mortalität der Umstand sehr mit ins Gewicht fallen, daß sicher ein größerer Prozentsatz der Fälle nicht veröffentlicht worden ist, in denen die Zwerchfellverletzung trotz operativen Verschlusses der Wunde mit dem Tode ausging, als dies für die glücklich verlaufenen Fälle zutrifft. Unser ungünstig verlaufener I. Fall ist sicher nicht seiner Zwerchfellverletzung erlegen. Denn die Zwerchfellwunde zeigte sich bei der Sektion solid verheilt, nirgends war ein Prolaps der Baucheingeweide $z \mathbf{u}$ bemerken, die Zwerchfellwölbung war links wiederhergestellt, der Zwerchfellstand war laut Sektionsprotokoll beiderseits gleichhoch. Das, was reichlich 3 Monate nach dem Unfall den Tod unseres Verletzten herbeigeführt hat, war die langdauernde Eiterung der Pleura in Verbindung mit den mehrfachen Abszedierungen der Bauchhöhle, denen der durch Lungenphthise obendrein noch geschwächte Körper nicht Widerstandskraft genug entgegenzusetzen hatte.

Was nun die Frage betrifft, ob der Zugang zur Zwerchfellwunde auf pleuralem oder abdominalem Wege genommen werden 
soll, so wird man wohl bei perkutane $\mathrm{n}$ Verletzungen sich von der äußeren Wunde den Weg weisen lassen müssen, d. h. man wird diese erweitern und verfolgen und so sich die Zwerchfellwunde zu Gesicht bringen. Anders bei subkutanen Verletzungen. Hier stehen sich verschiedene Ansichten gegenüber. Der eine Teil der Chirurgen gibt dem Operieren von der Bauchhöhle aus dem Vorzug, wohl zum Teil aus der alteingewurzelten Furcht heraus die Pleura zu eröffnen, weil man einerseits den Pneumathorax, andererseits die große Empfindlichkeit der Pleura gegen Infektion scheut, dann aber auch, weil man sagt, man könne auf diese Weise die so häufigen Nebenverletzungen der Abdominalorgane sicherer erkennen, der andere Teil operiert von der Pleura aus, weil von hier aus das Nähen des Diaphragma technisch ungleich bequemer ist, und behandelt auch von hier aus nach Möglichkeit die etwa vorhandenen Verletzungen der Abdominalorgane, eventuell nach Erweiterung der Zwerchfellwunde, wie dies z. B. Schlatter ${ }^{1}$ ), Dalton ${ }^{2}$ ), $\quad$ inni ${ }^{3}$ ), Prawdoljuboff ${ }^{4}$ ) u. a. taten.

Einige Chirurgen nun kombinieren beide Methoden, indem sie zunächst von der Pleurahöhle aus das Zwerchfell versorgen und alsdann die Laparotomie anschließen, um vorhandene intraabdominelle Verletzungen von hier aus zu behandeln. $\mathrm{Neu}$ $\mathrm{g}$ e b a u e r schlägt vor, nach der Absuchung der vom Bauch her sehr schlecht zu besichtigenden hochgelegenen Eingeweide von der Pleura aus stets zur Kontrolle tiefer gelegener Teile und übersichtlicher Reinigung des Bauchfells auch noch zu laparotomieren.

Nun, ob von vornherein, wenn man, wie bei subkutanen Verletzungen, die Wahl hat, transpleural oder transperitoneal operiert werden soll, darüber sind wohl die Akten geschlossen, nachdem sowohl $\mathrm{N}$ e u g e ba u e $\mathrm{r}$ wie $\mathrm{S} u$ ter statistisch festgestellt haben, daß die Mortalitätsstatistik bei den transpleural operierten ungleich besser ist, wie bei den transperitoneal behandelten. Suter berechnete für die ersteren eine Mortalität von 5,2 Proz. gegen eine solche von 28,5 Proz. für letztere, N e u -

I) Münchner med. Wochenschr. I90I, Nr. 34.

2) Journal of the Americ. med. Association I890; zit. nach Neugebauer l. c.

3) Ref. Zentralbl. f. Chir. 1894, Nr. 30.

4) Ref. Zentralbl. f. Chir. Igo8, Nr. 32. 
g e b a u e r fand 9,6 Proz. Mortalität bei ersteren, 5o Proz. Mortalität bei letzteren. Es is t d a herdertranspleuralen Methode, alsder MethodederWahl, unbedingt der Vorzug zu geben.

Nicht dagegen möchten wir den Standpunkt von Neugebauer teilen und bei jeder Zwerchfellverletzung die Laparotomie anschlieBen. Den an sich schwer Verletzten nun noch einen solchen zweiten schweren Eingriffohne $z$ wingenden Grund zuzumuten, halteich uns nicht fïr berechtigt.

Und in der Tat kann man ja auch mit ziemlicher Leichtigkeit die Organe, die eventuell verletzt sein könnten, durch die Zwerchfellwunde, eventuell nach Erweiterung derselben, vorziehen und gegebenenfalls von hier aus nähen. Wenigstens hat in unserem ersten Fall die Naht der beiden Perforationsstellen des Magens keinerlei Schwierigkeit bereitet, sehr zum Unterschied von der Naht der Zwerchfellwunde selbst, die zuweilen, je weiter man nach vorn an die vordere Thoraxwand herankam, unausführbar zu sein schien. Daß man in gewissen Fällen, in denen man von oben her nicht zurecht kommen kann, noch zur Laparotomie wird schreiten müssen, ist selbstverständlich.

Uber die Technik der Zwerchfellnaht brauchen wir keine Worte zu verlieren. Knopfnähte, durch die ganze Dicke des Zwerchfells hindurchgelegt, sind am bequemsten anzulegen und allgemein üblich. $\mathrm{Ob}$ Seide oder Katgut benutzt werden soll, das liegt im Ermessen des einzelnen Operateurs.

Soll nun die Pleurahöhle nach der Versorgung der Zwerchfellwunde ganz geschlossen oder drainiert werden?

Wenn eine Verunreinigung der Pleurahöhle mit Darm- oder Mageninhalt nicht stattgefunden hat, so ist der vollkommene Verschluß der Thoraxwunde wohl selbstverständliche Forderung, denn nur hierdurch regen wir die kollabierte Lunge wieder zur Entfaltung an und bringen wir den Pneumothorax zur raschen Resorption, wenn dagegen eine solche Verunreinigung stattgefunden hat, so könnte es von vornherein allzukühn erscheinen, wollte man auch hier die Pleurahöhle wieder vollkommen verschließers. 
Auch in unserem I. Falle führten diese Bedenken dazu, daß die Pleura durch Drainage offen gehalten wurde, und doch möchte ich glauben, daß es vielleicht nicht zu dem großen jauchenden Empyem gekommen wäre, wenn die Pleura auch in diesem Falle primär verschlossen worden wäre. I)ann hätte sich die Lunge entfalten können, und die durch den Pneumothorax nach Noetzels ${ }^{1}$ ) Untersuchungen verursachte Störung der Blutund Lymphzirkulation hätte nicht der Infektion der Pleura Vorschub leisten können.

Und sowie $R$ ehn ${ }^{2}$ ) es fürdie operative Behandlung der Lungenwunden gefordert hat, den. Thorax in jedem Falle nach Versorgung der L ungenwundezuverschlieBen, selbstauf dieGefahrhin, späterwiederöffnenzum üssen, um den Patientenersteinmal überdie Gefahr der mechanischen Zirkulationsstörungen hinwegzubringen, som öchte ich gla uben, da $B$ man genau diesen Grundsatz auch auf die Zwerchfellverletzungen übertragen muB.

Seit Erscheinen der Statistik Suters operierte Fälle von Zwerchfellverletzungen.

Rossi. (Clinica chirurgica I905, Nr. 4). Ref. Zentrabll. f. Chir. I906. Nr. I8, Fall von Stichverletzung des Zwerchfells mit Netzvorfall durch die durchbohrte Pleurahöhle und die Hautwunde. Zwerchfellnaht. Heilung.

E i c lue 1. (Deutsche med. Wochenschr. I906, Nr. 39.) Lanzenverletzung in der Höhe der rechten Io. Rippe. Bauchhöhle eröffnet. Loch im $/$ werchfell in der vordern Axillarlinie ca. $3 \mathrm{~cm}$ lang. Leber und Lunge sind nicht verletzt. Naht der Zwerchfellwunde. Heilung.

E i chel. (ibid.) Stichverletzung in der linken Seite. Äußere Wunde, kaum I cm groß, in der Axillarlinie oberhalb der 8. Rippe.

I) Archiv f. klin. Chir. I 906, Bd. 80 . Noet $z$ e l fand, daB Kaninchen eine künstliche Infektion der Pleura viel schwerer ertrugen, wenn ihnen ein Pneumothorax gesetzt wurde als ohne solchen und daß wiederum bei bestehendem Pneumothorax die Infektion schwerer verlief, wenr die Pleura durch Drainage offen gehalten als wenn sic geschlossen wurdc. Noet z e l crklärt sich diese Tatsache durch mechanische Zirkulationsstörungen im Bercich der Pleura, hervorgerufen durch clie Kompression der Lunge seitens des Pneumothorax.

2) Verhandlungen der deutschen Gescllschaft f. Chir. I905, S. I37. 
I82 WOLF, Über Zwerchfellverletzungen und ihre operative Behandlung.

Netzprolaps bis in die äußere Wunde. Loch im Zwerchfell etwa $2 \mathrm{~cm}$ lang. Erweiterung der Zwerchfellwunde, Absuchen des oberen Teiles der Bauchhöhle. Keine Verletzungen der Abdominalorgane. Naht der Zwerchfellwunde. Heilung.

An d rews. (Surgery, gynecol. and obstetr. Bd.4, H. 2.) Ref. Zentralbl. f. Chir. I907, Nr. 23. Stichwunde im 7. Interkostalraum durch Zwerchfell und Magen. Magenwunde vom Abdomen aus genäht. Naht derZwerchfellwunde von derBauchhöhle aus sehr schwierig, deshalb noch 2 Matratzennähte von außen durch den Interkostalraum. Heilung.

S s in a kew it s ch. (Russ. Archiv f. Chir. Igo6.) Ref. Zentalbl. f. Chir. I907, Nr. 23. Stichverletzung der linken Brustseite. $1 \frac{1}{2} \mathrm{~cm}$ lange Wunde im Zwerchfell, in die ein Stück Netz eingeklemmt ist. Resektion des Netzstückes. Abdominalorgane unverletzt. Naht des Zwerchfells. Tamponade der Pleura. Pleuraempyem, Heilung.

Ssinakewitsch. (ibid.) Schuß in die linke Brust. Einschuß zwischen den beiden Axillarlinien in Höhe der 7. Rippe. Zwerchfellwunde erweitert. Loch in der Magenwand (Mukosa unverletzt). Magen und Zwerchfell genäht. Tamponade der Pleura. Glatte Heilung

S s in a kew itsch. (ibid.) Schußverletzung. Einschuß links vorn. Das Geschoß sitzt im Angulus scapulae sin. unter der Haut. Nach 4 Tagen Resektion der 7. Rippe. Leicht blutiges, trübes Exsudat der linken Pleura. Das Zwerchfell steht hoch. Probepunktion ergibt stinkenden Eiter. Zwerchfell wird an die äußere Haut genäht und der Absze $B$ drainiert. Nach 3 Wochen wird beim Spülen ein ovales, glattrandiges Stück Leinewand ( $1 / 1 / 2 \mathrm{~cm}$ lang, I $\mathrm{cm}$ breit), das aus dem Hemd des Mädchens stammt, entleert, worauf Heilung.

Ssinakewitsch. (ibid.) Messerstich rechts zwischen 5. und 6. Rippe. Zwerchfell- und Leberverletzung. Leberwunde kauterisiert, Zwerchfellwunde nur teilweise genäht, Gazestreifen. Eitrige Pleuritis. Heilung.

$\mathrm{S}$ s in a kew its ch. (ibid.) Messerstich links. Erweiterung der Stichwunde, Tampon in die Pleura. Nach 3 Tagen stinkende Sekretion. Drainage. Exitus am 5 Tage. Sektion ergibt, daß ein Stück Magenwand in einer Zwerchfellwunde eingeklemmt und nekrotisch geworden ist.

Prawdoljuboff. (Chirurgia I908, Nr.36.) Ref. Zentralbl. f. Chir. I908, Nr. 32. 3 Stichverletzungen des Zwerchfells, 2 mal Netzprolaps in Hühnereigröße, einmal außerdem Verletzung des Colon transversum. Erweiterung der Zwerchfellwunden in allen 3 Fällen. Naht. Heilung.

$\mathrm{F}$ a s a n o. (XX. Italien. Chirurgenkongre 3 I907.) Ref. Zentralbl. f. Chir. I908, Nr. 40. Fall von penetrierender Zwerchfell- und Leberverletzung. Abnorm hohe Einstichöffnung im linken 5. Interkostalraum unterhalb der Mamilla. Lunge $\mathrm{n}$ i $\mathrm{h} t$ verletzt. Transpleurale Naht der Leberwunde. Befestigung der Wundränder des Zwerchfells an die äußere Wunde, um die Leberwunde tamponieren zu können. Heilung. 